\title{
Adjacency Matrix based Face Recognition Approach
}

\author{
Harish Rohil, PhD \\ Asst. Professor \\ Department of Computer Science and Applications \\ Chaudhary Devi Lal University, Sirsa, Haryana \\ (INDIA)
}

\author{
Pankaj Kaushik \\ M Tech Scholar \\ Department of Computer Science and Applications \\ Chaudhary Devi Lal University, Sirsa, Haryana
}

(INDIA)

\begin{abstract}
Biometric based techniques have emerged as the most promising option for recognizing individuals in recent years, for authenticating people and granting them access. Face recognition provide a biometric based authentication, authorization, surveillance services. Face recognition has gained increasing interest in the recent decade. Over the years there have been several techniques being developed to achieve high success rate of accuracy in the identification and verification of individuals for authentication in security systems. This paper proposes an adjacency matrix based approach for face recognition. The proposed approach was implemented in $\mathrm{C}$ and tested for different inputs.
\end{abstract}

\section{Keywords}

Face Recognition, Adjacency matrix, Image Processing, Edge Detection.

\section{INTRODUCTION}

Face recognition is achieved by means of comparing the rigid features of the face, which do not change over a period of time. It can be achieved by comparing other parameter such as skin tone against the information that is stored in face database. Many different algorithms are stored in the face database. Many different algorithms are already available to perform this comparison.

\subsection{Steps involved in Face Recognition}

Face recognition system (FRS) involves six basic steps [9] which are as follows:

a) Acquire: This step involves capturing the image containing the face. In case of $2 \mathrm{D}$ face recognition, a digital camera is needed. For 3D face recognition, an additional range camera is needed for obtaining the depth coordinates.

b) Detect: Face detection involves identifying the face in captured image and demarcating it from the image background.

c) Align: The face may not be completely perpendicular to the camera and hence the alignment needs to be determined and compensated for before recognition.

d) Extract: Face feature extraction involves a process of measuring various face features and creating a face template, face print, for the purpose of matching and identification. The parameters that can be used for creating the face template includes the following:

- Distance between the eye

- Contour of the nose

- Width of the nose

- Contour of the eye sockets
- Length of the jaw line

- $\quad$ Skin texture (skin print)

- Contour of the chin

e) Match: This involves the process of matching the $2 \mathrm{D}$ or 3D face template with the record in the face database. Matching often involves a scoring process. These scores are obtained by running the corresponding algorithms on the face dat. The higher scores indicate higher probability of the match being accurate.

f) Report: Reporting may involves returning one or more face matches depending on the usage scenario. Matches can be returned based on the score and user preferences.

\subsection{Face Detection}

Face Detection is a concept that includes many sub problems. Some systems detect and locate faces at the same time, others first perform a detection process and then [2], if possible, try to locate the face as shown in Fig: 1
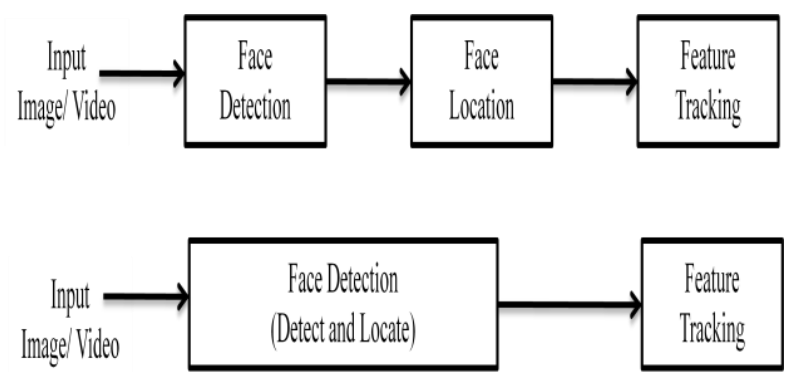

Fig 1: Face Detection Process of FRS

Face detection algorithms usually share common steps. Firstly, some data dimension reduction is done, in order to achieve an admissible response. Some pre-processing could also be done to adapt the input image to the algorithm prerequisites. Then, some algorithms analyze the image as it is, and some others try to extract certain relevant face regions. The next phase usually involves extracting face features or measurements. These will then be weighted, evaluated or compared to decide if there is a face and where is it. Finally, some algorithms have a learning routine and they include new data to their models.

Face detection is, therefore, a two class problem where we have to decide if there is a face or not in a picture. This approach can be seen as a simplified face recognition problem. Face recognition has to classify a given face, and there are as many classes as candidates. Consequently, many face detection methods are very similar to face recognition algorithms. 


\subsection{Feature Extraction}

The feature extraction process can be defined as the procedure of extracting relevant information from a face image. The feature extraction process must be efficient in terms of computing time and memory usage. The output should also be optimized for the classification step. Feature extraction involves several steps are - dimensionality reduction, feature extraction and feature selection Dimensional reduction is an essential task in any pattern recognition system. The process of feature extraction is shown in Fig- 2 below.

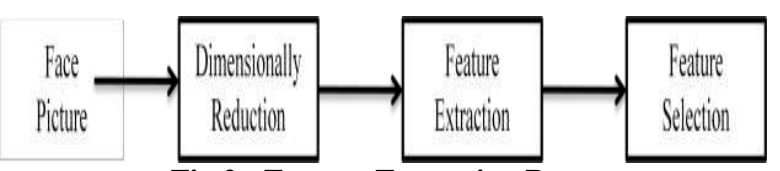

Fig 2: Feature Extraction Processes

Feature extraction and feature selection are two distinct steps. A feature extraction algorithm extracts features from the data. Feature selection is often performed after feature extraction. So, features are extracted from the face images, and then an optimum subset of these features is selected. The dimensionality reduction process can be embedded in some of these steps, or performed before them.

\subsection{Challenges in Face Recognition}

A face detection system, operating in real life scenarios, can be expected to encounter any of the challenges listed below [1]. For

Instance, a service robot and its supporting camera usually capture furniture, different light sources, and other objects along with humans.

a) Pose variations: Face image extracted for recognition may vary in pose styles like front view, side view etc. The image with different pose style make reorganization task very difficult.

b) Occlusions: Foreign objects obstructing the view of the camera make the task of detection (and recognition) difficult. Depending on the percentage of the face that is occluded, face detection may even be impossible.

c) Illumination: Another challenge is related to the illumination conditions under which the image was obtained. For instance, if the illumination was very low, the face contour might not be discernible from the background. Only a part of the face may be illuminated. Further, the source and direction of the illumination is also important.

d) Face components and structures: The presence or absence of beards and mustaches, changed hair styles is also challenges.

\section{RELATED WORK}

Many approaches have been proposed in the field of FRS, some of them have been discussed as follows:

Sarawat Anam et al. [4] have proposed an AI based system for FRS, after following 6 six basic steps of FRS, and then pass the extracted feature in Neural Network by using Back Propagation and genetic Algorithm. Features are extracted as follows:

$$
\mathrm{X}=\sum \mathrm{mx} / \sum \mathrm{m} \quad \mathrm{Y}=\sum \mathrm{mY} / \sum \mathrm{m}
$$

B. Vinay Kumar et al. [5] have proposed another AI FRS in which feature is extracted after image acquisition by using wavelet by specifying „Lower Bound (LB) “ and „Upper Bound (UB) " as following equation. Then image is recognized using network specifically using Multi layer Perceptron (MLP).

$$
\mathrm{F}_{\mathrm{LB}}=\frac{1}{\sqrt[x 1]{2}} \quad \mathrm{~F}_{\mathrm{UB}}=\frac{1}{\sqrt[x 2]{2}}
$$

Henry A. Rowley et al. [7] have proposed AI based system that operates on two stage: neural network based filter and then arbitrates the filter output- i) neural network based filter and ii) merging overlapping detections and arbitration. Neural network base filter: proposed system is a filter that receives as input a $20 \times 20$ pixel region of the image, and generates an output ranging from 1 to 1 , signifying the presence or absence of a face, respectively. To detect faces anywhere in the input, the filter is applied at every location in the image. Merging overlapping detections and arbitration: By setting a minimum threshold on the number of detections, much false detection can be eliminated. A second heuristic arises from the fact that faces rarely overlap in images. If one detection overlaps with another, the detection with lower confidence can be removed.

Steve Lawrence. et al [6] have proposed an system face recognition is by SOM (Self Organizing Maps), which are used to trained neural network from vectors which are extracted from image in first step of process. In proposed system SOM quantized 25 dimensional input vectors into 125 topological ordered values. After that convolution neural network is trained on new created training set. The method is capable of rapid classification, requires only fast, approximate normalization and preprocessing, and consistently exhibits better classification performance than the eigen faces approach.

Mihir Jain ET at. [10] have proposes whole process for Line Edge Map (LEM) based approach. First image is preprocessed by face detection, localization and orientation correction is done. After that eye detection is done by proposed algorithm. Eyes can be detected by feature based, appearance based methods, and template based methods. Eye Detection using LEM Template: Proposed algorithm is tolerated to some amount of background. The template used here is the LEM of an artificial pair of eyes including eyebrows. It is constructed according to the distance between the centroid with which it is to be matched. Edge map is obtained by Sobel method. Block wise and I- LEM Implementation: In this face LEM is divided into three blocks, containing eye region, nose region, and lip region. Then LEM of corresponding parts of each test face are compared with nose of each model face.

Vipin Kumar and Mohit Mehta [11] proposes the concept of an improved Line Edge Map (LEM) using Matlab has introduced and compared the results with famous recognition technique "eigenface" and with the earlier results of LEM using different algorithm. Firstly at the input we will give a RGB image then convert that RGB image into a grey scale image then grey scale image will be converted into binary image then using Sobel edge detection convert binary image into edge detected image then invert that edge detected image and apply improved LEM algorithm on it and we will get LEM image of the input image then compare that LEM image with data base and we will get the appropriate match if available. Fig: 2.1 shows proposed approach. 


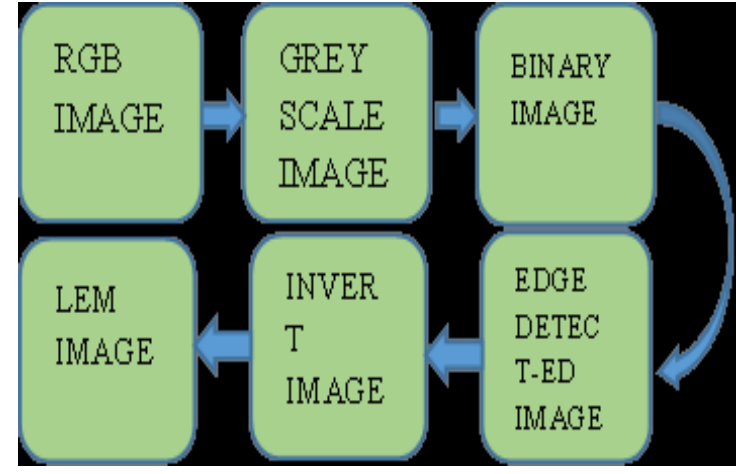

Fig 2.1: Block Diagram of Proposed Approach

\section{FUNDAMENTALS OF FACE RECOGNITION}

Many fundamental approaches used in face recognition. Some of them are discussed as follows:

1) Principal Component Analysis (PCA)

2) Linear Discriminant Analysis (LDA)

3) Artificial Neural Network (ANN) Based

4) Line Edge Map (LEM) Based

\section{1) Principal Components Analysis (PCA)}

The PCA technique converts each two dimensional image into a one dimensional vector. This vector is then decomposed into orthogonal principle components (known as eigen faces). In other words, the technique selects the features of the face which vary the most from the rest of the image. In the process of decomposition, a large amount of data is discarded as not containing significant information since $90 \%$ of the total variance in the face is contained in $5-10 \%$ of the components. This means that the data needed to identify an individual is a fraction of the data presented in the image [1]. Each face image is represented as a weighted sum (feature vector) of the principle components, which are stored in a one dimensional array. Each Eigen face represents only a certain feature of the face, which may or may not be present in the original image. A test image is compared against an image in database by measuring

The distance between their respective feature vectors.

\section{2) Linear Discriminant Analysis (LDA)}

LDA is a statistical approach based on the same statistical principles as PCA. LDA classifies faces of unknown individuals based on a set of training images of known individuals. The technique finds the underlying vectors in the face feature space (vectors) that would maximize the variance between individuals and minimize the variance within a number of samples of the same person [1]. If this can be achieved, then the algorithm would be able to discriminate between individuals and yet still recognize individuals in some varying conditions. To do this the algorithm must have an appropriate training set. The database should contain several examples of face images for each subject in the training set and at least one example in the test set. These examples should represent different frontal views of subjects with minor variations in view angle. As for PCA, LDA works well if the test image is relatively similar to the image in database in terms of size, pose, and illumination. With a good variety in sampling this can be somewhat varied, but only up to a point. For more significant variation other non-linear approaches are necessary.

\section{3) Neural Network Based Approaches}

The use of neural networks for face recognition by semisupervised learning method that uses support vector machines for face recognition. There have been many efforts in which in addition to the common techniques neural networks were implemented. In this, first dimensionality of face image is reduced by Principal component analysis (PCA) and later the recognition is done by the Back Propagation Neural Network (BPNN).

\section{4) Face recognition using Line Edge Map (LEM)}

Line Edge Maps (LEM) based approach proposes a line matching technique to make this task possible. LEM uses physiologic features from human faces in recognition [3]. It mainly uses mouth, nose and eyes as the most characteristic ones. In order to measure the similarity of human faces the face images are firstly converted into gray-level pictures. The images are encoded into binary edge maps using edge detection algorithm like Sobel, Difference of Gaussian (DoG) etc edge detection algorithm. The main advantage of line edge maps is the low sensitiveness to illumination changes, because it is an intermediate-level image representation derived from low-level edge map representation. The algorithm has another important improvement, it is the low memory requirements because the kind of data used. In Fig: 3, there is an example of a face line edge map; it can be noticed that it keeps face features but in a very simplified level.

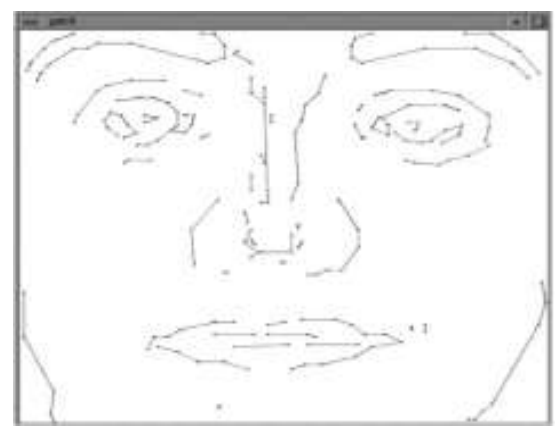

Fig 3: Example of face LEM

\section{PROPOSED ADJACENCY MATRIX BASED FACE RECOGNITION APPROACH}

This includes the design and implementation of adjacency matrix based algorithm that can be used for face recognition. Proposed algorithm is based on Line Edge Map (LEM) approach of a FERET. Proposed algorithm is implemented and tested by using programming language $\mathrm{C}$ turbo $\mathrm{C}++$ compiler.

\section{Algorithm 1: Proposed Algorithm for Acquired Image Preprocessing}

Proposed algorithm for acquired image preprocessing is used to process an input image before recognition is done. Same algorithm is used to populate and maintain database for many persons from whom recognition made from. Same algorithm is implemented on test image which is to be searched in database. Proposed algorithm is described in Fig: 4 below. 
I*Input: Image that is acquired from capturing devises */ /* Output: Line Edge Map of input image */

Begin:

1. Filtering: Remove all unusable background area from input image.

2. Cropping: Crop the filtered image to extract face from image

3. Edge Detection: Difference of Gaussian is applied to cropped image

End:

Fig 4: Proposed Algorithm for Image Processing

After LEM is produced form input image, now we convert LEM of image into adjacency matrix according to the following criteria.

Conversion of LEM in Matrix Form: After edge detection of input image, an adjacency matrix is generated from LEM. Adjacency matrix is generated on the bases of LEM, in which if edge is present in an particular grid, its value is detected as $\{1\}$, and those where no edge is found, its value is evaluated as $\{0\}$ as shown in Fig: 5. In the way every coordinates of grid view is studied and corresponding $\{0\}$ or $\{1\}$ is detected and adjacency matrix corresponding to every input is produced.
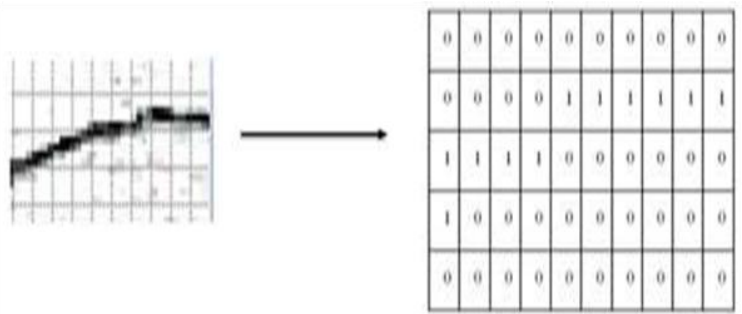

Block of Line Edge Map

\section{Matrix Generated} from Shown LEM

Fig 5: LEM to Matrix Conversion

After generating LEM for all images, and supplying this LEM for every image in database as image reference for face recognition along with other personal information, database is maintained.

\begin{abstract}
Algorithm- 2: Proposed Algorithm for Face Recognition This proposed algorithm is used in face recognition process. This algorithm is applied to test image, which is to be searched in database, and also to each and every image of person in database, after applying image processing algorithm. This algorithm describes the criteria for feature matching between two images.
\end{abstract}

Feature Matching: In this step, every image adjacency matrix in database is compared with test image (i.e. which is to be searched). If $\{1\}$ is found in both matrix on sane coordinates of matrix then this is called as Feature Match.
Variable Declaration: Before describing algorithm for face recognition some variables that are used in recognition is discussed as below:

1. Feature Match Count (C): All corresponding feature match is added up in an integer variable, which is incremented by 1 , on every match.

2. Threshold $\left(\mathbf{C}_{\mathbf{T h}}\right)$ : Threshold value is the minimum feature match value which is acceptable in recognition process. Threshold value is declared on the bases of matrix size of LEM. Larger the size of matrix larger will be the value of Threshold

Criteria for Recognition: Image with maximum $\left(\mathbf{C}_{\mathbf{F}}\right)$ is a recognized image in database called as Output of system. If $\mathbf{C}$ (for every image) is less than $\mathbf{C}_{\mathbf{T h}}$, than results in unrecognized person i.e. person is not found in database. Proposed algorithm for face recognition is described in Fig: 6 below.

/* Input: Adjacency matrix of image which is to be recognized */

I* Output: Result is displayed as Recognized or

Unrecognized person $* /$

Begin:

1. Calculate $C_{F}$ for all images in database.

2. Compare $\mathrm{CF}$ of each image in database.

3. Select Maximum $\mathrm{C}_{\mathrm{F}}$ value.

4. Compare selected $\mathrm{C}_{\mathrm{F}}$ with $\mathrm{C}$

If $\left(\mathrm{C}_{\mathrm{F}}>\mathrm{C}_{\mathrm{F}}\right)$

Then, selected CTh valued image is recognized image

Else if $\left(\mathrm{C}_{\mathrm{TH}}<\mathrm{C}_{\mathrm{F}}\right)$

Then, test image is unrecognized image.

End

Fig 6: Proposed Algorithm Face Recognition

\subsection{Populating Database}

In this section we will populate our database with different persons, on which we will test our proposed work. While populating, we need one image of a person according to which person is to be identified, and some sort of details about the same person which is displayed as output to give information about identified person.

a) Image Data 1: In this we populate our database with the present image of Pankaj Kaushik as shown in Fig: 7 (a). On this input image firstly proposed image processing algorithm. 


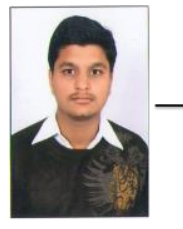

Input

Image

(Pankaj

Kaushik)
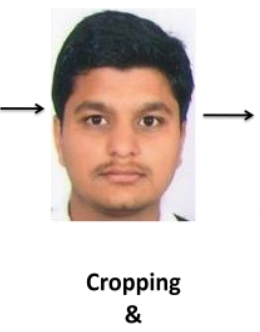

Filtering

(b)

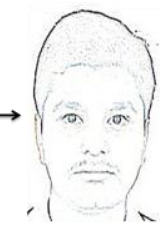

Edge

Detection

(c)

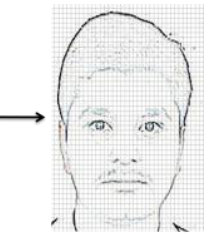

Grid

View

(d)
Fig 7: Image Processing Algorithm Applied to Populate image of Pankaj Kaushik

As explained, firstly input image filtered and cropped to extract only face image with eliminating useless background space which is shown in Fig:7 (b). After extracting full face image, Difference of Gaussian(DoG) operation is applied to Line Edge Map (LEM) of face image as shown in Fig:7 (c). By viewing LEM in grid view an adjacency matrix is generated as shown in Fig: 8 (b). This matrix is passed as an image identity of Pankaj Kaushik in database.

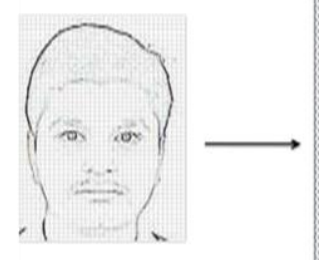

Line Edge Map

(a)

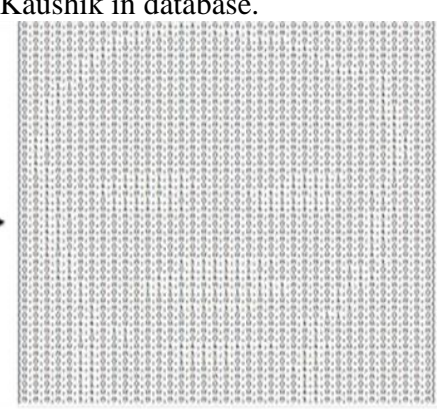

Adjacency Matrix

(b)
Fig 8: Conversion LEM to Adjacency Matrix for Data1

b) Image Data 2

In this section we populate our database with the image of Dr. Harish Rohil as shown in Fig: 9(a). Same proposed image processing algorithm is applied to that image.

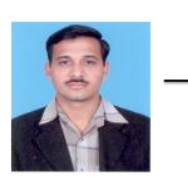

Input
Image
(Dr. Harish
Rohil Rohil
(a)

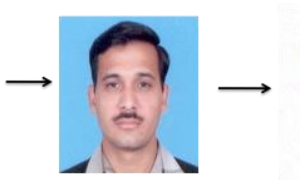

Cropping Filtering

(b)

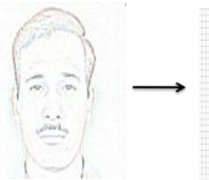

Edge Detection

(c)

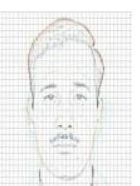

Grid View

(d)
Fig 9: Image Processing Algorithm Applied to Populate image of Dr. Harish Rohil

First of all input image filtered and cropped to extract only face image with eliminating useless background space which is shown in Fig:9 (b). After extracting full face image, Difference of Gaussian (DoG) operation is applied to Line Edge Map (LEM) of face image as shown in Fig: 9(c). By viewing LEM in grid view an adjacency matrix is generated as shown in Fig: 10(b). This matrix is passed as an image identity of Dr. Harish Rohil in database.

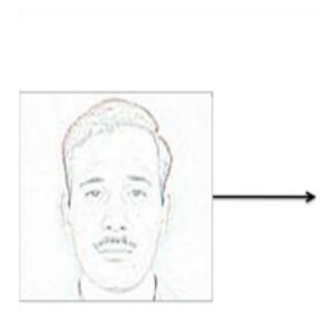

Line Edge Map

(a)

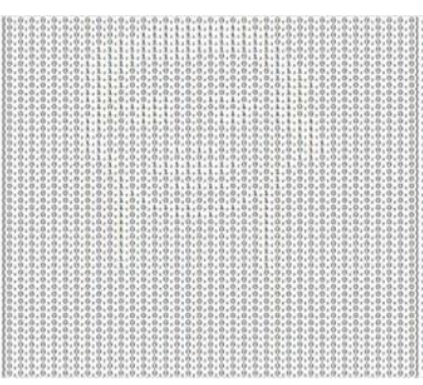

Adjacency Matrix

(b)
Fig 9: Conversion LEM to Adjacency Matrix for Data 2

\subsection{Experiment 1}

Experiment 1 is performed on an image with the goal to test whether person in test image is present in our database or not. a) Test Image Processing: In this section a test image of comedian Kapil Sharma is passed to verify the proposed approach for face recognition. First input test image is processed to extract adjacency matrix from it. Input image of Kapil Sharma (shown in Fig:10 (a)) is cropped to get exact face image which is shown in Fig: 10(b).

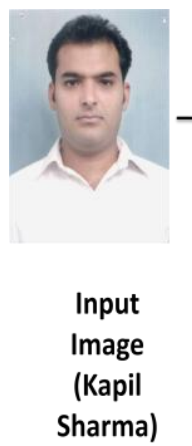

(a)
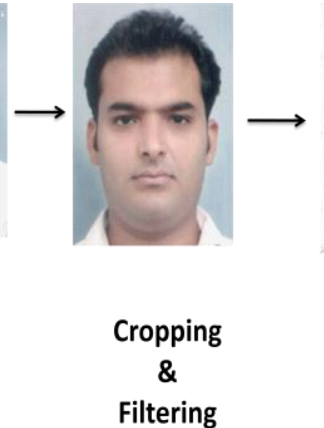

(b)

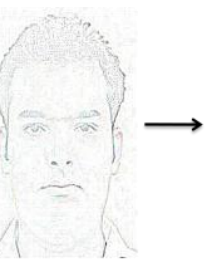

Edge Detection

(c)

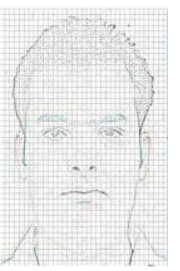

Grid View
Fig 10: Image Processing on Test Image of Kapil Sharma in Experiment 1

After cropping LEM is extracted by DoG edge detection as shown in Fig: 10(c). After then, by viewing in grid view, an adjacency matrix is generated (as shown in Fig: 11(b)), which is passed in coding to check for person in database.

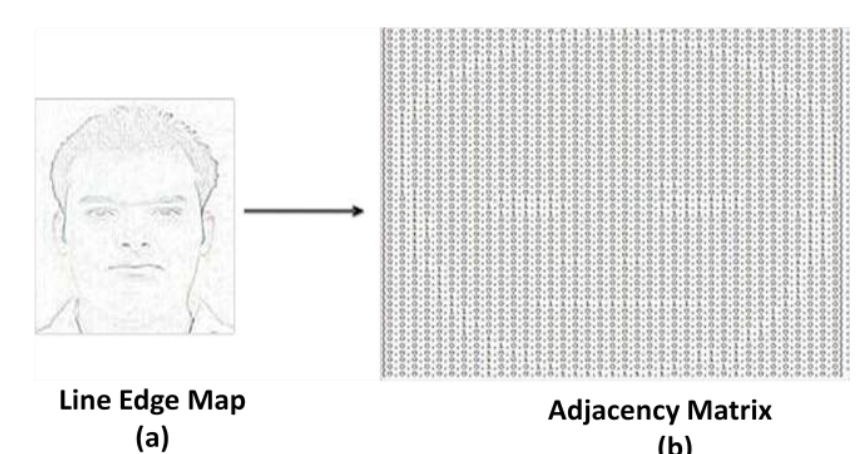

Fig 11: Conversion of LEM to Adjacency Matrix of Test Image

\section{Face Recognition Result}


After passing adjacency matrix of test image of Kapil Sharma in program and after proceed recognition by proposed approach, we will try to identify the person in our database. As we know our database contain data of Pankaj Kaushik and Dr. Harish Rohil, so our desired output will be as unidentified person. The output of program is shown in Fig: 12, which is an snapshot of turboc $\mathrm{C}++$ console output screen. Thus proposed approach is verified successfully.

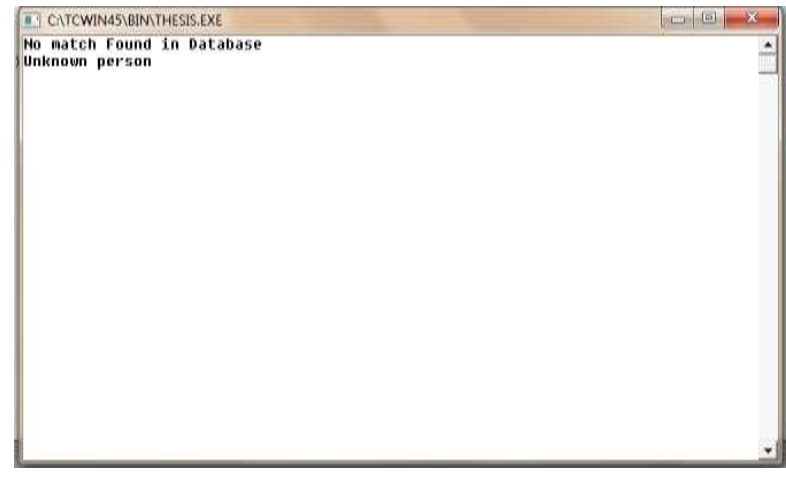

Fig 12: Output after Passing Test Image for Recognition in Experiment 1

\subsection{Experiment 2}

Experiment 2 is performed on another input image with the same goal to test whether person in test image is present in our database or

Not.

a) Test Image Processing: This time a six year earlier image of Pankaj Kaushik, is passed as test image in proposed system to verify our approach. First input test image is processed to extract adjacency matrix from it. Input image of Pankaj Kaushik (shown in Fig: 13 (a)) is cropped to get exact face image which is shown in Fig: 13(b).

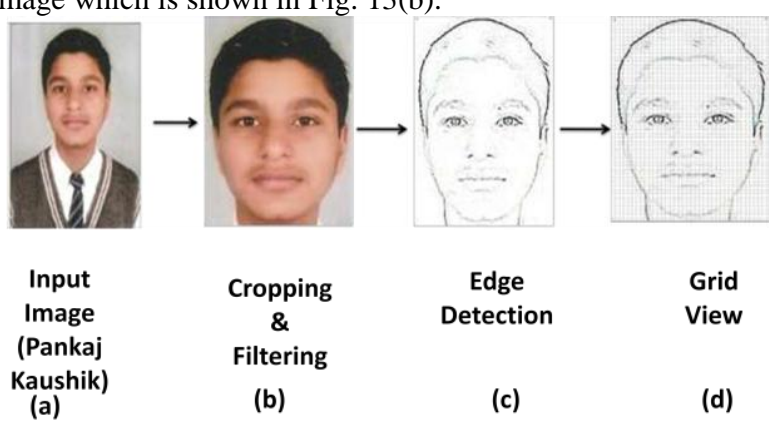

Fig 13: Image Processing on Test Image of Pankaj Kaushik in Experiment 2

After cropping LEM is extracted by DoG edge detection as shown in Fig: 13(c). After then, by viewing in grid view, an adjacency matrix is generated (as shown in Fig: 14(b)), which is passed in coding to check for person in database.

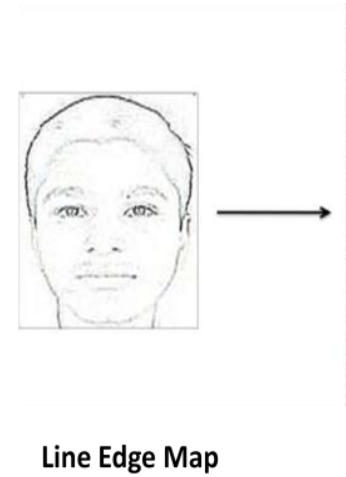

(a)

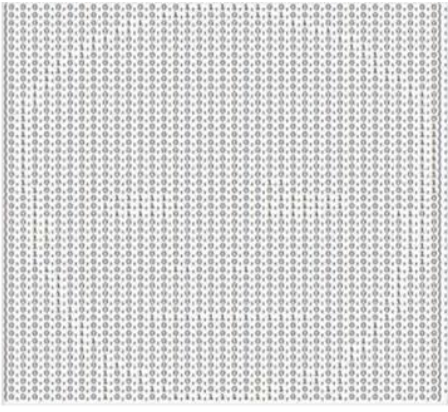

Adjacency Matrix

(b)
Fig 14: Conversion of LEM of Test Image into Matrix Form in Experiment 2

\section{Face Recognition Result}

After passing adjacency matrix of test image of Pankaj Kaushik in program and after following recognition by proposed approach, we will try to identify the person in our database. As we know our database contain data of Pankaj Kaushik and Dr. Harish Rohil, our desired output will be as identified person as Pankaj Kaushik and other related information about the same is displayed. The output of program is shown in Fig: 15, which is a snapshot of turboc $\mathrm{C}++$ console output screen. Thus proposed approach is verified successfully.

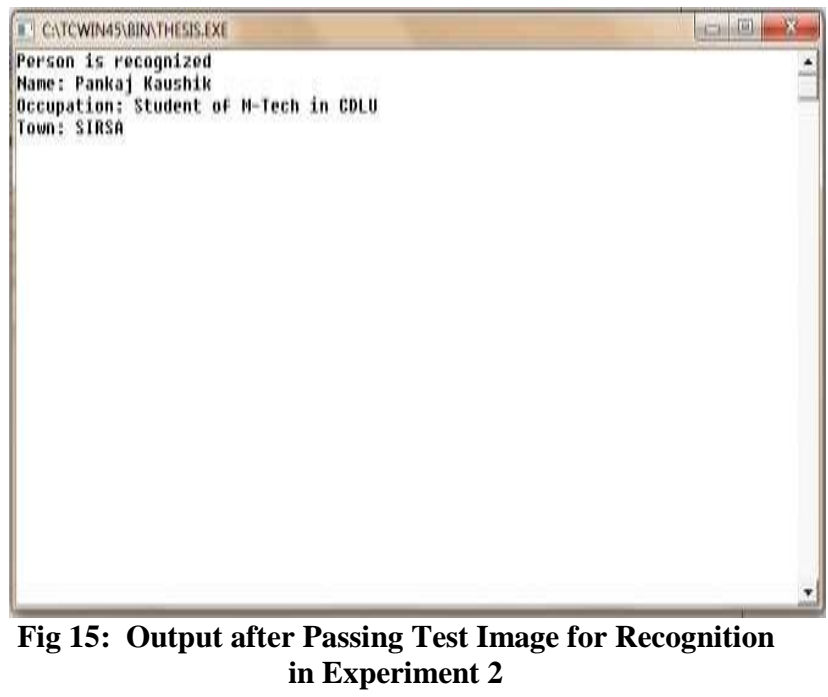

\section{CONCLUSION}

This paper has proposed an adjacency matrix based approach for face recognition. The paper includes generic steps of face recognition, describing face detection process of recognition system. The various challenges faced by face recognition system have also been discussed. This paper also include fundamental of face recognition such as PCA, LDA, AAN, LEM. In this paper we proposed image processing algorithm and face recognition algorithm, which is implemented using $\mathrm{C}$ language. From the proposed work we have concluded that proposed algorithm for face recognition is much better as compared to others techniques in error occurring conditions like lighting conditions, pose variations, various face expression. Our proposed matrix based Line Edge Map (LEM) approach is effective in every condition and capable of achieving high accuracy as compared with other techniques. 
The advantages of proposed approach are- less time consuming, less memory occupying and faster technique. This approach can be implemented by an individual with less expertise knowledge. These algorithm can easily be implemented using various image processing tools, which in hole make it an easily applicable approach in the field of face recognition. The proposed approach is verified by.

\section{REFERENCES}

[1] Lucas Introna, Helen Nissenbaum, Face Recognition Technology, New York University 2009

[2] Ion Marques, Proyecto Fin de Carrera, Face Recognition Algorithms, June 16, 2010.

[3] Jorge Orts, Recognition Techniques, the University of Wisconsin.

[4] Sarawat Anam,Shohidul. et al, Face Recognition Using Genetic Algorithm and Back Propagation Neural Network", International Multi Conference of Engineers and Computer Scientist, Vol I, 2009.
[5] B. Vinay Kumar, et al, A Back Propagation Based Face Recognition Model, Using 2D Symmetric Gabor Features, IEEE ISBN: 1-4244-0997-7/07/\$25.

[6] Steve Lawrence. Et al, Back Face Recognition: A Convolution Neural Network Approach, IEEE Volume 8, Number 1, pp.98-113, 1997.

[7] Henry A. Rowley. et al, Neural Network Based Face Detection" Computer Vision and Pattern Recognition, 1998.

[8] O T Arulogun.et al "Development of Security System using Face Recognition", Computer Science and Engineering Dept., Ladoke Akintola University of Technology, Nigeria

[9] Atul Gupta . Face Recognition, White Paper, Infosys.

[10] Mihir Jain ET at Eye Detection and Face Recognition using Line Edge Map, DA-IICT:

[11] Vipin Kumar, Mohit Mehta, Punjab College of Engineering Face Recognition using Line Edge Map, International Journal of Multidisciplinary and Current Research, ISSN: 2321-3124. 